\title{
Preparation of silicon-based nanowires and the thermochemistry of the process
}

\author{
D. Hourlier ${ }^{*}$, P. Lefebvre-Legry ${ }^{*}$, P. Perrot ${ }^{\$}$ \\ * Institut d'Electronique, de Microélectronique et de Nanotechnologies, \\ UMR-CNRS 8520, F-59652 Villeneuve d'Ascq \\ djamila.hourlier@iemn.univ-lille1.fr, patricia.lefebvre@iemn.univ-lille1.fr \\ \$ Laboratoire de Métallurgie Physique et Génie des Matériaux, UMR-CNRS 8517, \\ Université des Sciences et Technologies de Lille, F-59655 Villeneuve d'Ascq \\ pierre.perrot@univ-lille1.fr
}

\begin{abstract}
The present paper is part of a general investigation of the thermodynamic properties of metal-semiconductor systems involved in the nanowires growth. Here, we will show that many aspects of the growth mechanisms, such as the surface curvature of the solid phase (in the nano or micrometer range), in equilibrium with the liquid phase are extremely important. We calculate and construct all binary Au-Si phase diagrams corresponding to nanosystems. By choosing the $S(V) L S$ process rather than the VLS process, we succeeded in answering not only the origin of the driving force needed to trigger off the precipitation of solid nanowire but also the thorny question of the size limit of nanowires in relation with our specific growth conditions.
\end{abstract}

\section{Keywords:}

Silicon nanowires, VLS mechanism, thermodynamics, phase diagrams, nanosystems.

\section{INTRODUCTION}

The synthesis of semiconductor nanowires has recently attracted much attention for their potential applications in electronic, photonic and biological areas [1]. Hence, many efforts have been devoted to developing different approaches for synthesizing a variety of semiconductor nanowires. However, Silicon nanowires are much desirable because of the central function that silicon plays in the current semiconductor processing technology. The most extensively synthesis method used is based on the vapour phase technique with Au particles as mediating solvent. The vapour phase of the desired material can be generated through various sources: either the decomposition of gaseous precursors such as $\left(\mathrm{SiH}_{4}, \mathrm{SiCl}_{4}, \mathrm{Si}_{2} \mathrm{H}_{6}\right)$ or the thermal evaporation of condensed phases. In these techniques, the generally accepted mechanisms of the nanowire growth are referred to the vapour-liquid-solid (VLS) process when the precursor is gaseous or Solid-Liquid-Solid (SLS) process when the starting material to be evaporated is solid. Whatever the process used, the solid nanowire is formed by precipitation from a supersaturated droplet alloy (Auconstituent of the growing material). The diameters of the nanowires are dictated by the size of the alloy droplet considered as a template. In the VLS process in which silane gas acts as Si feedstock, it is easy to understand the origin of supersaturation of gold droplet as the silicon chemical potential in gaseous phase is much higher relative to silicon substrate. This contrasts with the SLS process. Actually, in the SLS process, silicon nanowires are grown by heating the metal-coated silicon substrate (wafer) at high temperature in an atmosphere of argon and hydrogen. The only source of silicon is "apparently" the solid silicon wafer.

Based on the bulk Au-Si phase diagram, many researchers [2-4] postulated that the growth of nanowires by the SLS mechanism occurred through silicon diffusion from the substrate to the gold-silicon melt formed on the surface at high temperatures. With time, more silicon from the substrate diffuses into the droplet, making it supersaturated with silicon and then the coexisting pure silicon phase precipitates and crystallizes as nanowires. The same interpretation has been used to explain the growth of nanowires on other metals: $\mathrm{Ni}, \mathrm{Pt}, \mathrm{Pd}, \mathrm{Al}, \mathrm{Fe}$ [5-7]. However, no explanation was given to justify the supersaturation.

From a thermodynamic point of view, it is easy to accept the saturation of gold with silicon. However, once the saturation is obtained, the chemical potential of silicon in solid, liquid alloy (metal-Si) and gaseous phases is the same, and there is no thermodynamic (energetic) reason for the silicon of the wafer to supersaturate the binary liquid phase.

The true mechanism had never been proposed, neither species involved nor have the most important reaction pathways for nanowire growth been investigated. Systematic data which would be helpful for elucidation of the growth mechanism of nanowires, as well as information concerning the binary Au-Si diagram for the catalytic nanosystem, are practically unavailable in the literature.

A better understanding of the nanowire growth process in the vapor phase is needed to elucidate the growth mechanism and to be able to rationally control their compositions, sizes, crystal structures, and growth directions.

Thus, the purpose of the present work is to discuss, from a thermodynamic point of view coupled with experimental work, the growth of nanowires by the SLS process and to point out the conditions in which nanowires can be obtained.

\section{EXPERIMENTAL PART}

Successful synthetic strategy has been developed to obtain nanowires. Single-side polished n-type silicon wafers (111) (Siltronix) were used as substrates for subsequent Gold deposition. The wafers were first cleaved and then cleaned using a standard RCA process. The substrates were placed into a vacuum chamber, where $5 \mathrm{~nm}$ thick layer of gold was thermally evaporated onto their polished surface. The synthesis chamber consists of a horizontal tube (quartz or Alumina for high temperatures) heated by a three-zone furnace, with gas introduced into one end and pumped out

This is an Open Access article distributed under the terms of the Creative Commons Attribution-Noncommercial License (http://creativecommons.org/licenses/by-nc/3.0/), which permits unrestricted use, distribution, and reproduction in any noncommercial medium, provided the original work is properly cited. 
the other. For the SLS process, the experiments were carried out under a dynamic gas atmosphere $(3 \% \mathrm{H} 2$ in nitrogen: 99.995 purity) at a flow rate of $50 \mathrm{~cm} 3 / \mathrm{min}$. The gold coated wafers were annealed up to $1100^{\circ} \mathrm{C}$, with the heating rate of $10^{\circ} \mathrm{C} / \mathrm{min}$.

The as-synthesized nanowires are characterized and analyzed by field emission scanning electron microscopy Ultra 55 ZEISS (FE-SEM) and transmission electron microscope (TEM) (JEOL 2010 at $200 \mathrm{kV}$ ).

\section{RESULTS AND DISCUSSION}

\subsection{Phase diagrams}

To understand the origin of silicon-based nanowires growth, it is necessary to back off a bit and consider the interaction between $\mathrm{Au}$ and $\mathrm{Si}$. Therefore, we begin by examining the Au-Si phase diagram by taking into account the possible equilibria between a solid particle and a liquid droplet. The radius of the liquid droplet is not necessarily identical to that of the solid. In the usual Au-Si phase diagrams both the solid and liquid phases are either macroscopic or nanoscopic with the same shape and size $[8,9]$. The macroscopic phase diagram in which both phases are larger than $100 \mathrm{~nm}$ does not correspond to the system involved in nanowire growth. In fact, at the beginning of the $\mathrm{S}(\mathrm{V}) \mathrm{LS}$ process, the solid is macroscopic (wafer substrate), that is, in equilibrium with a liquid in nano-meter range; then, after precipitation of silicon and the start of the nanowire growth, we may consider a new system in which a nanoscopic solid (nanowire) is in equilibrium with a nanoscopic liquid (alloy droplet). Therefore, it should be emphasized that attention must be paid to all these systems where the liquid phase is in the nanometer range whereas the solid phase is either macroscopic or nanoscopic in size. Moreover, if the solid phase is in the nanometer range, we have to distinguish between a nanometric solid whose shape is spherical and the nanowires whose shape is cylindrical.

The Au-Si phase diagrams are calculated by using the CALPHAD method (CALculation of PHAse Diagrams). This is based on finding the minimum total Gibbs energy of the system at constant temperature and pressure, yielding the composition and the amount of phases in equilibrium. The liquid solutions are modelled using the Redlich-Kister polynomial.

In figure 1 the calculated phase diagrams of typical systems involved during the growth process of nanowires are plotted. For the sake of simplicity, only three metastable systems are represented and compared with the best known stable system, in which the liquid and the solid are both in the macroscopic range. When the liquid is nanometric, it is supposed to be spherical with $5 \mathrm{~nm}$ radius. When the solid is nanometric, it is supposed to consist of spherical nanocrystals of $5 \mathrm{~nm}$ radius or cylindrical nanowires of $5 \mathrm{~nm}$ radius.

The phase diagrams in figure 1 are all of the same shape. Gold forms a simple and deep eutectic system with silicon. However, the eutectic temperature and the eutectic composition depend on the solid/liquid system considered. It must be pointed out that the calculations are made under the implicit hypothesis that the surface tension does not depend on the particle size. Such an assumption has been proved valid [10] for nanoparticles whose radius exceeds 4 $\mathrm{nm}$. For smaller particles, surface tension decreases and tends towards zero when the diameter of the particle tends towards zero.

The most important new information provided in figure 1 is that the silicon solubility depends not only on the size of the liquid droplet but also on the shape and size of the

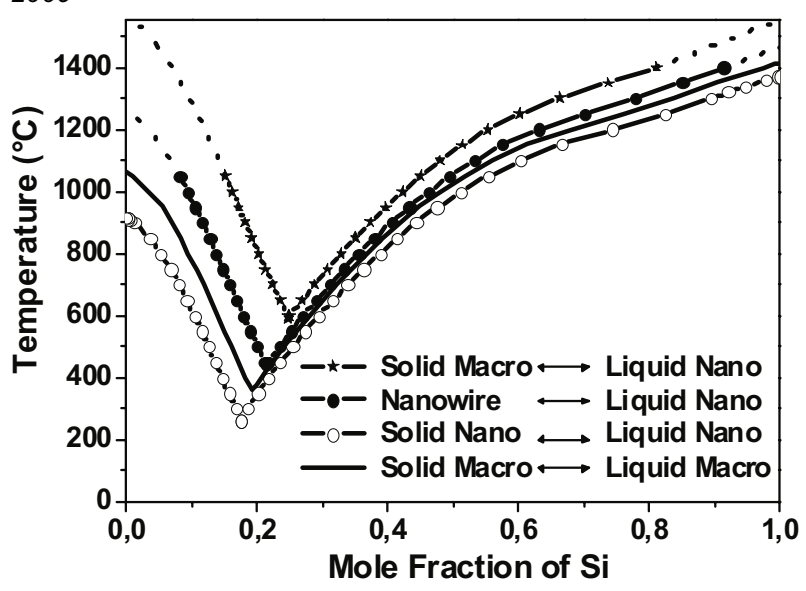

Figure 1: The effects of the size and shape of the solid and liquid phases on $\mathrm{Au}-\mathrm{Si}$ phase diagrams (stable and metastable systems).

solid in equilibrium with that liquid. This calculation will be extended to illustrate an important point, namely, the melting behaviour of gold particles and the nature of the solid surface (substrate) on which the nucleation of nanowires has occurred. This kind of analysis is in progress; however, it is beyond the scope of the present study.

With the variety of cases encountered in this study, we are now in a position to treat a liquid alloy (Au-Si) of any size in equilibrium with any silicon-based solid (nano or macroscopic size).

These diagrams also afford an opportunity for verifying descriptions of other related synthesis methods (CVD, GSMBE...) by the VLS process.

Valuable studies on the growth of nanowires by the VLS mechanism have been reported by many researchers [11] who refer to the macroscopic Au-Si system. However, the difference in the eutectic temperature of macroscopic system and the one corresponding to small gold particles in equilibrium with the solid substrate shows that knowledge of the bulk system alone is not sufficient to permit the evaluation of systems in nanometric range.

Thermodynamically, however, these diagrams show significant differences in liquidus temperatures and not fundamental differences in the nature of the components. Thus, the condensed phases present in the system show that there is no convenient driving force coming from the wafer to justify any supersaturation of the gold nanodroplet with silicon of the substrate. Hence, particular attention will be paid on Si present in the gas phase.

\subsection{SLS process: Phase gas analysis}

Si wafers coated thick Au film were heated at $1000^{\circ} \mathrm{C}$ for 1 h. figure 2 (A) shows the SEM image of the Au deposited on Si wafer, heated for $10 \mathrm{~min}$. At this stage the Au film on the Si wafer breaks up and nanoparticles with diameters in the range 10-100 $\mathrm{nm}$ were formed on the Si surface. These nanoparticles act as the nucleation site for the growth of Sibased nanowires figure $2 \mathrm{~B}$

In the SLS mechanism, $\mathrm{Au}, \mathrm{Si}$ and $\mathrm{Ar}$ (with or without $\mathrm{H}_{2}$ ) are the main elements introduced in the synthesis chamber. Another element to be considered is oxygen which arises either from leakage, from degassing of $\mathrm{Al}_{2} \mathrm{O}_{3}$ tube or simply from $\mathrm{SiO}_{2}$ which may be native or even intentionally grown by thermal deposition on the substrate. 

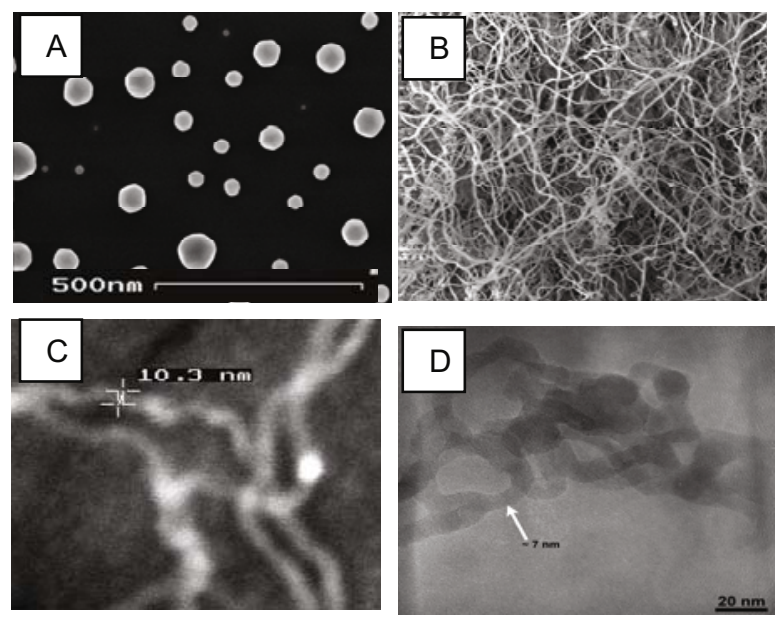

Figure 2: The catalytic growth sequences: A-gold particles after few minutes heating

A thermochemical analysis was made of the reduction and volatilization which occur in the $\mathrm{Si} / \mathrm{O}$ system. Hence, the silicon species present in the gas phase may be $\mathrm{Si}, \mathrm{Si}_{2}, \mathrm{Si}_{3}$ in equilibrium with the solid silicon, or $\mathrm{SiO}$ in equilibrium with the $\mathrm{Si}-\mathrm{SiO}_{2}$ mixture.

Figure 3 shows log $P_{\mathrm{SiOx}}$ vs $P_{\mathrm{O} 2}$ plots of the data. The vertical lines in the figure give the oxygen pressures for the oxidation reaction:

$$
\mathrm{Si}(\mathrm{s})+\mathrm{O}_{2} \leftrightarrows \mathrm{SiO}_{2}(\mathrm{~s}) \quad[\mathrm{A}]
$$

The most important feature in figure 3 is the $\mathrm{SiO}(\mathrm{g})$ pressure peak at the interface $\mathrm{Si}(\mathrm{s})-\mathrm{SiO}_{2}(\mathrm{~s})$. The main equilibrium to be considered is thus the following one:

$$
\mathrm{Si}(\mathrm{s})+\mathrm{SiO} 2(\mathrm{~s}) \leftrightarrows 2 \mathrm{SiO}(\mathrm{g})
$$

$R T \ln \left(P_{\mathrm{SiO}} / P^{\circ}\right)^{2}=-\Delta_{\mathrm{rB}} \mathrm{G}^{\circ}+R T \ln \left(\mathrm{a}_{\mathrm{Si}} \times \mathrm{a}_{\mathrm{SiO} 2}\right)$

$\Delta_{\mathrm{rB}} \mathrm{G}^{\circ}=718614.749-532.537892 T+24.4690697 T \ln T$ $+4.99486782 \times 10^{-4} T^{2}+187.917772 \times 10^{-9} T^{3}-923$ $266.15 T^{-1}$

The coefficients are extracted from [12].

The standard state for $\mathrm{SiO}_{2}$ is the solid cristobalite, which is the stable form of silica under the temperature range of interest. As seen above, the standard state for $\mathrm{Si}$ is the pure solid silicon $<\mathrm{Si}$, dia $>$; the standard pressure for gaseous species $\left(\mathrm{O}_{2}\right.$ and $\left.\mathrm{SiO}\right)$ is $P^{\circ}=10^{5} \mathrm{~Pa}$.

If the wafer $\left(a_{S i}=1\right)$ is in equilibrium with the solid cristobalite $\left(a_{\mathrm{SiO} 2}=1\right)$, the calculated partial pressure of $\mathrm{SiO}$ is $4.05 \mathrm{~Pa}$ at $1100^{\circ} \mathrm{C}$. When the equilibrium is reached, the chemical potential of silicon is the same in every phase and no more driving force exists to justify the nanowire growth experimentally observed. The explanation of this process has to be researched in the presence of metastable equilibria developed in the system.

The only source of metastability comes from the fact that $\mathrm{SiO}_{2}$ on the wafer is amorphous silica rather than crystallized under the form of stable cristobalite, as it can be easily shown by X-ray diffraction on the silica layers. Actually, all deposited and thermally grown oxides in semiconductor process are amorphous.

Thus, the activity of the amorphous silica deposited on the silicon wafer would be:

$$
a_{\mathrm{SiO} 2, \mathrm{am}}=P_{\mathrm{SiO} 2, \mathrm{am}} / P_{\mathrm{SiO} 2, \mathrm{crist}}^{\circ}>1
$$

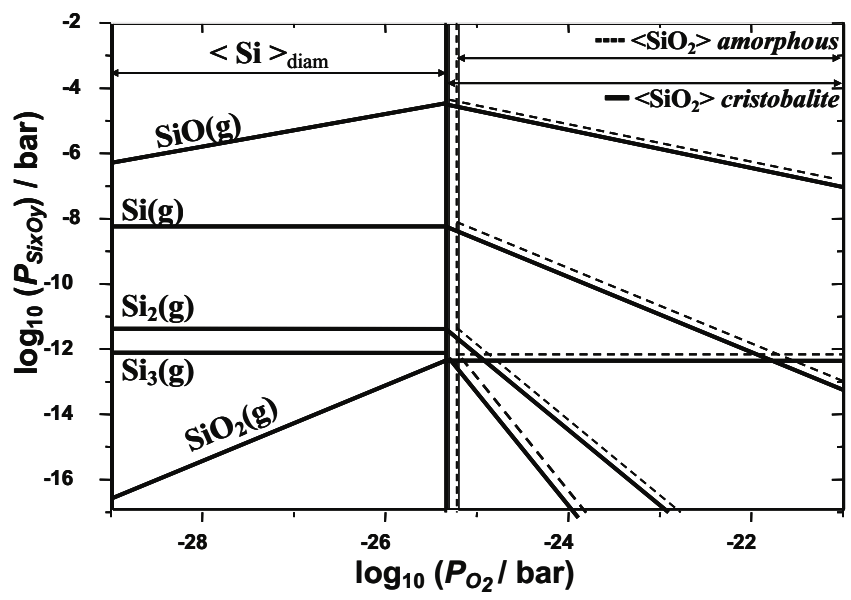

Figure 3: Thermochemical data for Si-O system at $1373 \mathrm{~K}$.

The partial pressure of SiO developed by the equilibrium [B] would be higher above the $\mathrm{Si} / \mathrm{SiO}_{2}$ (amorphous) couple than above the $\mathrm{Si} / \mathrm{SiO}_{2}$ (cristobalite) couple. The activity of amorphous silica, $a_{\mathrm{SiO} 2,}$ am is calculated from the transformation:

$$
\begin{aligned}
& \left.\left.<\mathrm{SiO}_{2}\right\rangle_{\text {crist }} \leftrightarrows<\mathrm{SiO}_{2}\right\rangle_{\text {am }} \\
& \Delta_{\mathrm{rC}} \mathrm{G}^{\circ}=\mu_{\mathrm{SiO} 2, \mathrm{am}}^{\circ}-\mu^{\circ}{ }_{\mathrm{SiO} 2, \mathrm{crist}}=-R T \ln a_{\mathrm{SiO} 2, \mathrm{am}}
\end{aligned}
$$

$\Delta_{\mathrm{rC}} \mathrm{G}^{\circ}=21951.202-156.202 T+22.12745 T \ln T-$ $147.0421 \times 10^{-4} T^{2}+1775.6603 \times 10^{-9} T^{3}-$ $1584843.057 T^{-1}$

$\mu^{\circ}{ }_{\mathrm{SiO} 2, \mathrm{am}}$ is obtained by extrapolation of $\mu^{\circ}{ }_{\mathrm{SiO} 2, \mathrm{~L}}$ towards low temperatures. At $1100^{\circ} \mathrm{C}$ :

a $\mathrm{SiO} 2, \mathrm{am}=1.267$ and $\left(P_{\mathrm{SiO}}\right)$ calculated from equation 3 and related to the system

$\mathrm{Si} \mathrm{dia} / \mathrm{SiO} 2 \mathrm{am}=4.562 \mathrm{~Pa}>\left(P_{\mathrm{SiO}}\right)$ at equilibrium $\mathrm{Si}$ $\mathrm{dia} / \mathrm{SiO} 2 \mathrm{crist}=4.05 \mathrm{~Pa}$

$$
P_{\mathrm{SiO}}=K_{1} \sqrt{a_{\mathrm{Si}} a_{\mathrm{SiO}_{2}}}
$$

This difference brings the driving force needed to supersaturate the gold droplets with $\mathrm{Si}$. Gaseous $\mathrm{SiO}$ above the wafer, stable with respect to the $\mathrm{Si} / \mathrm{SiO} 2$ (amorphous) mixture, is thus metastable with respect to the $\mathrm{Si} / \mathrm{SiO} 2$ (cristobalite) mixture, and the reaction [B] will naturally proceed towards the decomposition of SiO. Without gold droplets, $\mathrm{Si}$ and $\mathrm{SiO} 2$, resulting from the disproportion reaction

$2 \mathrm{SiO} \rightarrow \mathrm{SiO}_{2}+\mathrm{Si}$, will germinate on the wafer. The gold droplets acting as a catalyst, $\mathrm{SiO}$ will decompose on the surface of the droplet and silicon will dissolve until the liquid-gas equilibrium is reached. When the liquid is in equilibrium with the gaseous phase but supersaturated in silicon with respect to the wafer, the precipitation of silicon nanowires will be observed. Amorphous $\mathrm{SiO}_{2}$ synthesized by the disproportion reaction will deposit on the nanowire and on the wafer.

\subsection{The minimum size of nanowires}

The driving force of the silicon nanowire growth $\Delta_{d} G$ is the chemical potential of silicon in the droplet in equilibrium with the gas phase and that of silicon wafer:

$\Delta_{\mathrm{d}} G=\mu_{\mathrm{Si}}$, supersaturation $-\mu^{\circ} \mathrm{Si}$, saturation $=\mu_{\mathrm{Si}}$, gas $-\mu^{\circ} \mathrm{Si}$, diam 
$\Delta_{\mathrm{d}} G=2 R T \ln \left[P_{\mathrm{SiO}}(\mathrm{Sidiam} / \mathrm{SiO} 2 \mathrm{am}) / P_{\mathrm{SiO}}(\mathrm{Si} \mathrm{diam} / \mathrm{SiO} 2 \mathrm{am})\right]$

The Gibbs energy of the transformation of Si (diamond) into $\mathrm{Si}$ (nanowire) via $\mathrm{SiO}$ is related to the radius of nanowire by the following equation: $\Delta_{\mathrm{d}} G=\sigma V / r_{\text {min }}$

$r=\left[R T \ln \left(P_{\mathrm{SiO} \text { nano/am }} / P_{\mathrm{SiO} \text { dia/am }}\right)\right]=4.5 \mathrm{~nm}$

The nanowire growth energy increases when $r$ decreases, cannot be higher than the available energy $\Delta_{d} G$, a result which implies the existence of a minimum radius $r_{\text {min }}$

Thus, what we labelled the first generation of nanowires have their radius higher or equal to $r_{\min }=4.5 \mathrm{~nm}$ at $1100^{\circ} \mathrm{C}$ as confirmed by SEM analysis (figure $2 \mathrm{~B}$ and $\mathrm{C}$ ).

The question then arises as to how nanowires (firstgeneration) are affected by their thermal surrounding. In other words: How do nanowires behave upon the constraints imposed by the system?

The relationship between activities of solids and pressures of gases resulting from the reaction $(A)$ is expressed by:

$K(T)=\left(P_{\mathrm{SiO}}\right)^{2} /\left[a_{\mathrm{Si}} \times a_{\mathrm{SiO} 2}\right]=\exp \left(-\Delta_{\mathrm{r}} G^{\circ} / R T\right)(4)$

$K(T)$ is the equilibrium constant closely related to the Gibbs energy $\Delta_{\mathrm{r}} G^{\circ}$ of the reaction (A) and depends only on the temperature. It will be recalled that the superscript $\left({ }^{\circ}\right)$ refers to all components of the reaction being in their standard states that is $\mathrm{Si}, \mathrm{SiO}_{2}$ and $\mathrm{SiO}$ under the diamond, cristobalite and gaseous form at $0.1 \mathrm{MPa}$ pressure, respectively.

Once the first-generation of nanowires are grown, a new phase which is metastable silicon nanowire, is formed. Amorphous $\mathrm{SiO}_{2}$ is still present as a metastable phase and the $\mathrm{SiO}$ pressure developed in the system is no more $P_{1}=$ 4.562 $\mathrm{Pa}$ but a new pressure $P_{2}=5.212 \mathrm{~Pa}$ which is generated by the equilibrium $<\mathrm{Si}$, nanowire $>/<\mathrm{SiO}_{2}$, amorphous $>$. While $K(T)$ has a fixed value, the equilibrium pressures of the individual components do not. Thus there is an infinite number of sets of pressures of reactants and products which satisfy equation [4] and which correspond to equilibrium states of the system. $P_{2}$, the $\mathrm{SiO}$ pressure at the $\mathrm{Si}$ nanowire/SiO${ }_{2}$ amorphous equilibrium and $P_{0}$, the $\mathrm{SiO}$ pressure at the $\mathrm{Si}$ diamond/ $/ \mathrm{SiO}_{2}$ cristobalite equilibrium are computed from $K$.

It should be noted that such pressure change of $\mathrm{SiO}$ from $P_{1}$ to $P_{2}$ occurs according the vaporisation reaction of $\mathrm{Si}$ nanowires and $\mathrm{SiO}_{2}$. The vaporisation of the first-generation of nanowires supplies enough energy to proceed with the growth of the next generation of nanowires. The free energy imposes a new size limit of nanowires. The corresponding minimum radius of the second-generation of nanowires is calculated by the same relation (5): $\quad r=\sigma_{\mathrm{Si}} V_{\mathrm{Si}} / \Delta_{\mathrm{d}} G=$ $2.39 \mathrm{~nm}$

The minimum value of $r$ decreases when $P_{\mathrm{SiO}}$ increases, thus, nanowires characterized by $r<4.78 \mathrm{~nm}$ must be generated by a SiO pressure $P_{2}>P_{1}$.

Taking into account the lack of precision of the numerical values, it is possible to say that the minimum diameter of the second generation nanowires is half of the diameter of the first- generation nanowires. The second generation nanowires are rarer than those of first- generation owing to the difficulty in obtaining liquid nanodrops with a radius of the order of $2.5 \mathrm{~nm}$. The great majority of droplets have a radius higher than $2.5 \mathrm{~nm}$ and it is hard to imagine the formation of a nanowire whose diameter would be much lower than the droplet size. However, the process involving the vaporization of the first-generation of nanowires will tend to cause the radius of nanowires to decrease whereby the $\mathrm{SiO}$ pressure is high enough to allow the growth of the next generation of nanowires whose size limit has been estimated to be near $2.5 \mathrm{~nm}$.
The subsequent generation (third, fourth...) of silicon nanowires growth proceeds in the same way as before in the cycle considered previously.

The calculation is confirmed by TEM observations (Figure 2D). The process is considered as a permanent repetition of the cycles by growth and vaporization of the wires. By this process, there is theoretically no minimum size for the nanowires.

\section{SUMMARY}

Our experimental results are in full agreement with our thermodynamics predictions. In detail, the $S(V) L S$ is used to fabricate a composite $\mathrm{Si} / \mathrm{SiO} 2$ nanowires. The present paper evaluates the case of $\mathrm{Si}-\mathrm{Au}$, but the principles should be sufficiently general to hold for other binary alloys as well.

\section{Acknowledgments}

This work was supported by the Centre National de la Recherche Scientifique (CNRS) grant No 949 IPAM.

\section{REFERENCES}

[1] Ozin, G, Arsenault, A, Nanochemistry: A chemical approach to nanomaterials, RSC 2005, Printed by Henry Ling Limited, at the Dorset Press, UK.

[2] Yan, H, F, Xing, Y, J, Hang, Q, L, Yu, D, P, Wang, Y, $\mathrm{P}, \mathrm{Xu}, \mathrm{J}, \mathrm{Xi}, \mathrm{Z}, \mathrm{H}$, Feng, S, Q, 2000, Growth of amorphous silicon nanowires via a solid-liquid-solid mechanismChem. Phys, Lett, 323, 224-228.

[3] Yu, D. P, Xing, Y. J, Hang, Q, L, Yan, H. F, Xu, J, Xi, Z. H, S.Q. Feng, S.Q, 2001, Controlled growth of oriented amorphous silicon nanowires via a solidliquid-solid (SLS) mechanism Physica, E9, 305-309.

[4] Paulose, M, Varghese, O, K, Grimes, C, A, 2003, Synthesis of Gold-Silica Composite Nanowires

Through Solid-Liquid-Solid Phase Growth, J. Nanosci. Nanotech, 3, 341-346.

[5] Carter, D, J, Qu, Y, Porter, R, Hoang, L, Masiel, D, J, Guo, T, 2005, Silicon-based nanowires from silicon wafers catalyzed by cobalt nanoparticles in a hydrogen environment, Chem. Commun, 2274-2276.

[6] Chang, J, B, Liu, J, Z, Yan, X, Bai, L, F, Yan, Z, J, Yuan, X, M, Yang, Q, 2006, Ultrafast growth of singlecrystalline Si nanowires, Mater. Lett. 60, 2125-2128.

[7] Park, H.K, Yang, B, Kim, S.W, Kim, G.H, Hyeb Youn, D.H, Kim, S.H, Maeng, S.H, 2007, Formation of silicon oxide nanowires directly from $\mathrm{Au} / \mathrm{Si}$ and $\mathrm{Pd}-\mathrm{Au} / \mathrm{Si}$ substrates, Physica E 37 158-162.

[8] Tanaka, T, Hara, S, 2001, Thermodynamic Evaluation of Binary Phase Diagrams of Small Particle Systems, Z. Metallkde. 92(5), 467-472.

[9] Vallée, R, Wautelet, M, Dauchet, J.P, Hecq, M, 2001 Size and segregation effects on the phase diagrams of nanoparticles of binary systems, Nanotech, 12, 6874.

[10] Samsonov, V, M, Sdobnyakov, N, Y, Bazulev, A, N, 2004, Size dependence of the surface tension and the problem of Gibbs thermodynamics extension to nanosystems, Colloids Surf. A:Physicochem. Eng. Aspects, 239, 113-117.

[11] Schmidt, V, Gösele, U, 2007, How Nanowires Grow, Science, 316, 698-699.

[12] Schunurre, S, M, Gröber, J, Schmid-Fetzer, R, 2004, Thermodynamics and phase stability in the Si-O system, J.Non-Cryst. Solids, 336, 1-25. 